\title{
Li-S 电池硫正极性能衰减机理分析及研究现状概述
}

\author{
刀岩* 谢凯* 洪晓斌熊仕昭 \\ (国防科学技术大学 航天科学与工程学院 长沙 410073)
}

\begin{abstract}
摘要 由于汽车工业的持续发展, 对高能量密度二次电池的需求逐步增加, 锂硫电池开始走进人们的视野. 锂硫电池 的理论比能量高达 $2600 \mathrm{Wh} / \mathrm{kg}$, 而单质硫的理论比容量达 $1680 \mathrm{mAh} / \mathrm{g}$. 同时, 硫的储量丰富, 廉价, 并且环境友好. 虽 然可充电锂硫电池相比于传统锂离子电池有诸多优势, 但目前其可实现的实际比容量远低于理论比容量, 循环寿命也 较短等弊端限制了其大规模应用. 作者从 Li-S 电池正极的工作原理出发, 对硫正极容量损失及衰减机理做了深刻的解 析, 并结合本实验室的工作归纳总结了导致硫正极容量衰减的主要因素. 针对硫正极容量衰减因素, 从碳导电结构、聚 合物包覆以及纳米金属氧化物添加剂等方面，对近年来提高硫正极性能的主要研究方向及最新研究进展进行了综述， 并对其中存在的问题进行分析，最后对提高 Li-S 电池的整体性能提出展望.
\end{abstract}

关键词 Li-S 电池; 硫正极; 容量衰减; 碳导电结构; 聚合物包覆; 纳米金属氧化物添加剂

\section{Analysis of the Sulfur Cathode Capacity Fading Mechanism and Review of the Latest Development for Li-S Battery}

\author{
Diao, Yan* Xie, Kai* Hong, Xiaobin Xiong, Shizhao \\ (National University of Defense Technology, College of Aerospace Science and Engineering, Changsha 410073)
}

\begin{abstract}
Because of automotive industry sustainable development, the demand for higher energy density rechargeable batteries make the lithium-sulfur ( $\mathrm{Li}-\mathrm{S}$ ) batteries become one of the most attractive candidates. The Li-S systems have a theoretical specific energy of $2600 \mathrm{Wh} / \mathrm{kg}$ while the theoretical capacity of sulfur is $1680 \mathrm{mAh} / \mathrm{g}$. Sulfur is abundant, low cost and environment friendly. Although the rechargeable Li-S batteries possess more advantages over the conventional lithium ion batteries, the practical use faces with a variety of problems such as low specific capacity and short cycle life. Based on the fundamental electrochemical process of the sulfur cathode, the capacity fading mechanism of the sulfur cathode is analyzed in details. Combining with the works of our research team, the factors leading to the cathode property fading mechanism are summarized. Firstly, the main issue is that sulfur is both ionically and electrically insulating. And the insoluble low-order lithium polysulfide discharge products are also expected to be electronic insulators. So the cathode structure must contain electronic conductors (carbon or metal powder) which will decrease the energy density. Secondly, researchers impute the capacity fading into the residual $\mathrm{Li}_{2} \mathrm{~S}_{2}$ and $\mathrm{Li}_{2} \mathrm{~S}$ in sulfur cathode even at $100 \%$ depth of charge. The formation of $\mathrm{Li}_{2} \mathrm{~S}_{2}$ and $\mathrm{Li}_{2} \mathrm{~S}$ increasing with cycling results in active material loss. And the deposition of irreversible $\mathrm{Li}_{2} \mathrm{~S}$ or $\mathrm{Li}_{2} \mathrm{~S}_{2}$ at cracked surfaces of carbon particles causes cathode structural failure. Thirdly, high ordered lithium polysulfide $\left(\operatorname{Li}_{2} \mathrm{~S}_{n}, 3 \leqslant n \leqslant 8\right)$ is soluble in electrolyte, but low ordered lithium polysulfide $\left(\mathrm{Li}_{2} \mathrm{~S}_{2}\right.$ and $\left.\mathrm{Li}_{2} \mathrm{~S}\right)$ is insoluble. Thus chemical precipitation/dissolution reactions occur during the electrochemical process resulting in active material transition between liquid phase and solid phase. But it is difficult for the high ordered lithium polysulfide to transfer completely from liquid phase to solid phase at the end of cycles, so that will lead to the active material loss. Fourthly, another serious problem is the irreversible oxidation of cathode active material. The formation of $\mathrm{Li}_{x} \mathrm{SO}_{y}$ species increasing with cycling indicates an important capacity fading mechanism of Li-S battery. In this paper, the main research directions and the latest development to enhance the performance of sulfur cathode are reviewed from the aspects of carbon conductive structure, polymer coatings and metal oxides additives, and also the problems in each research directions are analyzed. Finally, the further development of Li-S battery is discussed. Keywords Li-S battery; sulfur cathode; capacity fading; carbon conductive structure; polymer coatings; nano-metal oxides additives
\end{abstract}

\section{1 引言}

随着社会高速发展对能源需求量的与日俱增, 以及 化石燃料对环境的污染和储量的枯竭, 使得人们对新型 替代能源的需求日益迫切. 传统锂离子电池(LIB)作为 新型替代能源而被广泛研究, 但由于其能量密度很难突
破 $300 \mathrm{Wh} / \mathrm{kg}$ ，限制了其在混合动力交通工具(PHEV)及 纯电动交通工具 $(\mathrm{PEV})$ 等领域的广泛应用. 电动交通工 具若要实现 300 英里(500千米)的运行距离, 所需电池的 能量密度要达到 $550 \mathrm{Wh} / \mathrm{kg}$, 因此大型高能量密度、低 成本的可再生能源系统开始逐步走入人们的视野 ${ }^{[1,2]}$. 由于单质硫密度轻、价格低廉、环境友好以及高比容量,

*E-mail: diaoyandiaoyan@sina.com; xie_kai@hotmail.com

Received December 10, 2012; published February 1, 2013 
被认为是一种优良的锂电池正极材料. 以单质硫作为正 极, 金属锂作为负极的 Li-S 电池, 理论能量密度高达 $2500 \mathrm{Wh} / \mathrm{kg}$ (体积能量密度可达 $2800 \mathrm{Wh} / \mathrm{L}$ ). 与传统的 锂离子电池相比, 能够实现其 $3 \sim 5$ 倍的能量密度 ${ }^{[3,4]}$, Li-S 电池因而备受关注.

\section{2 硫正极工作原理}

Li-S 电池的工作原理示意图见图 1. 硫正极的电化 学反应包括多步骤氧化还原反应，同时伴随着硫化物的 复杂相转移过程 ${ }^{[5]}$. 在放电过程中, 固相单质硫 $\mathrm{S}_{8(\mathrm{~s})}$ 首 先溶解在电解液中形成液相单质硫 $\mathrm{S}_{8(1)}$, 然后按照反应 方程(2) (6)逐步被还原 ${ }^{[6]}$. 方程式(1) 是单质硫的溶解 平衡方程式. $\mathrm{S}_{8(1)}$ 由于在第二个电化学还原反应中被消 耗, 导致固相单质硫进一步溶解. 电解液中的 $\mathrm{S}_{8(1)}$ 逐步 被还原成中间产物 $\mathrm{S}_{n}^{2-}(4 \leqslant n \leqslant 8)$, 聚硫离子与锂离子相 结合形成长链聚硫锂, 它们易溶于电解液, 并将从正极 结构中向电解液中扩散. 随着放电深度的加深, 长链聚 硫离子进一步被还原, 生成低价态聚硫离子 $\mathrm{S}_{2}^{2-}$ 和 $\mathrm{S}^{2-}$, 与锂离子结合发生沉淀反应, 生成在电解液中的溶解度 极低的 $\mathrm{Li}_{2} \mathrm{~S}_{2}$ 和 $\mathrm{Li}_{2} \mathrm{~S}$, 如方程式(7)和(8). 在充电过程中, 放电产物 $\mathrm{Li}_{2} \mathrm{~S}_{2}$ 和 $\mathrm{Li}_{2} \mathrm{~S}$ 逐步被氧化成长链聚硫锂, 最终 被氧化为单质硫. 如果单质硫按照反应方程(1)至(8) $100 \%$ 转化为 $\mathrm{Li}_{2} \mathrm{~S}$, 则单质硫的理论放电比容量可达 $1680 \mathrm{mAh} / \mathrm{g}$.

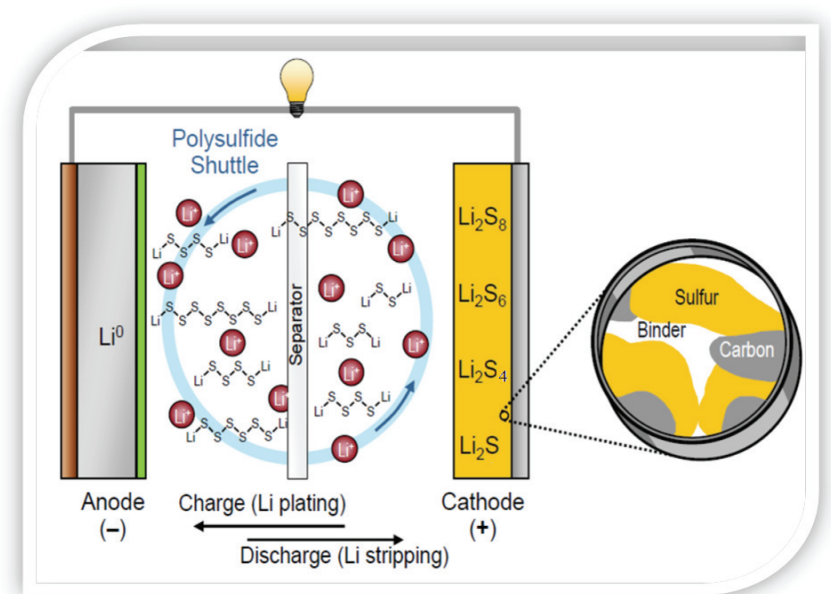

图 1 Li-S 电池工作原理示意图

Figure 1 Schematic operation proposed for the rechargeable Li-S battery

$$
\begin{aligned}
& \mathrm{S}_{8(\mathrm{~s})} \Leftrightarrow \mathrm{S}_{8(1)} \\
& \frac{1}{2} \mathrm{~S}_{8(1)}+e \Leftrightarrow \frac{1}{2} \mathrm{~S}_{8}^{2-} \\
& \frac{3}{2} \mathrm{~S}_{8}^{2-}+e \Leftrightarrow 2 \mathrm{~S}_{6}^{2-}
\end{aligned}
$$

$$
\begin{aligned}
& \mathrm{S}_{6}^{2-}+e \Leftrightarrow \frac{3}{2} \mathrm{~S}_{4}^{2-} \\
& \frac{1}{2} \mathrm{~S}_{4}^{2-}+e \Leftrightarrow \mathrm{S}_{2}^{2-} \\
& \frac{1}{2} \mathrm{~S}_{2}^{2-}+e \Leftrightarrow \mathrm{S}^{2-} \\
& \mathrm{S}_{2}^{2-}+2 \mathrm{Li}^{+} \Leftrightarrow \mathrm{Li}_{2} \mathrm{~S}_{2} \downarrow \\
& \mathrm{S}^{2-}+2 \mathrm{Li}^{+} \Leftrightarrow \mathrm{Li}_{2} \mathrm{~S} \downarrow
\end{aligned}
$$

Li-S 电池放电曲线的形式取决于聚硫离子的存在 形态. Li-S 电池具有两个典型的放电平台, 如图 2 所示. 通常高电压平台的电压从 $2.45 \mathrm{~V}$ 降至 $2.1 \mathrm{~V}$, 对应硫正 极的电极反应 $(2) \sim(4)$. 低电压平台的电压维持在 $2.1 \sim$ $1.7 \mathrm{~V}$, 对应硫电极反应 $(5) \sim(6)$.

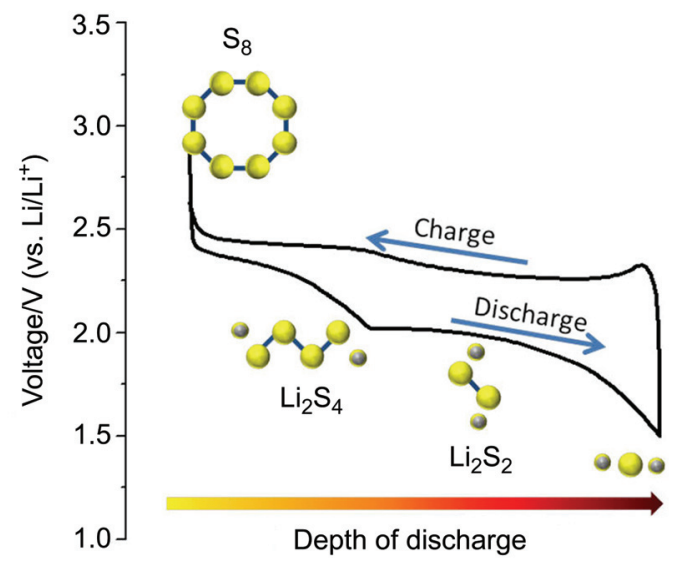

图 2 Li-S 电池充放电曲线原理示意图 ${ }^{[4]}$

Figure 2 Discharge-charge property of Li-S battery ${ }^{[4]}$

高电压放电平台总反应方程式可以简化为式(9), 及其对应的 Nernst 方程为式(10):

$$
\begin{aligned}
& \mathrm{S}_{8(1)}+4 e \Leftrightarrow 2 \mathrm{~S}_{4}^{2-} \\
& E_{\mathrm{H}}=E_{\mathrm{H}}^{\Theta}+\frac{R T}{n_{\mathrm{H}} F} \ln \frac{\left[\mathrm{S}_{8(1)}^{0}\right]}{\left[\mathrm{S}_{4}^{2-}\right]^{2}}
\end{aligned}
$$

低电压放电平台总反应方程式可以简化为式(11), 及其对应的 Nernst 方程为式(12):

$$
\begin{aligned}
& \mathrm{S}_{4}^{2-}+4 e \Leftrightarrow 4 \mathrm{~S}^{2-} \\
& E_{\mathrm{L}}=E_{\mathrm{L}}^{\Theta}+\frac{R T}{n_{\mathrm{L}} F} \ln \frac{\left[\mathrm{S}_{4}^{2-}\right]}{\left[\mathrm{S}^{2-}\right]^{4}}
\end{aligned}
$$

在高电压放电平台阶段，随着放电深度的逐步加 深, $\mathrm{S}_{4}^{2-}$ 的浓度逐渐增加, 然而由于单质硫的低溶解度 
$(0.019 \mathrm{~mol} / \mathrm{L})$, 溶液中单质硫的浓度基本保持在硫的饱 和浓度 $S_{8(1)}^{0}$ 或低于饱和浓度值, 而相比于 $S_{4}^{2-}$ 的浓度变 化, 几乎可以忽略不计, 所以 Nernst 方程(10)中, 高电 压平台的电压 $E_{\mathrm{H}}$ 受 $\mathrm{S}_{4}^{2-}$ 的浓度变化影响较大, 呈逐渐降 低的趋势. 在低电压平台阶段, $\mathrm{S}^{2-}$ 的浓度始终保持在 饱和浓度值, 而 $\mathrm{S}_{4}^{2-}$ 的浓度随着放电的进行逐渐降低, 但是由于这是一个十分缓慢的动力学过程, $\mathrm{d}\left(\left[\mathrm{S}_{4}^{2-}\right]\right)$ 随 $\mathrm{d} t$ 变化很小, 所以放电曲线电压在较长时间内基本保持 在 $2.1 \sim 2.0 \mathrm{~V}$, 直到 $\mathrm{S}_{4}^{2-}$ 的浓度降低到一定程度, 电压才 会出现急剧降低, 到达反应终止 ${ }^{[7]}$.

实际上, 在 Li-S 电池中, 硫活性物质的转化过程并 不是严格按照上述方程式(1) (8)逐步进行的, 具体反 应更加复杂, 这是由聚硫离子本身特性所决定的 ${ }^{[8]}$. 例 如聚硫锂在放电过程中会发生歧化反应, 如方程式 (13) (14), 而这些反应有利于最终放电产物的生成.

$$
\begin{aligned}
& \mathrm{Li}_{2} \mathrm{~S}_{n}+2 e+2 \mathrm{Li}^{+} \rightarrow \mathrm{Li}_{2} \mathrm{~S} \downarrow+\mathrm{Li}_{2} \mathrm{~S}_{n-1} \\
& x \mathrm{Li}_{2} \mathrm{~S}_{n-1} \rightarrow \mathrm{Li}_{2} \mathrm{~S} \downarrow+y \mathrm{Li}_{2} \mathrm{~S}_{n}
\end{aligned}
$$

长链聚硫锂能够与负极金属锂发生如方程式(15) (16)所示的还原反应, 并且长链聚硫锂也能够与不溶的 $\mathrm{Li}_{2} \mathrm{~S}_{2}$ 和 $\mathrm{Li}_{2} \mathrm{~S}$ 发生如方程式(17) (18) 所示的还原反应. 这些反应的存在使得 $\mathrm{Li}-\mathrm{S}$ 电池中存在一种特殊的效 应一飞梭效应. 硫正极生成的长链聚硫锂, 由于浓度 梯度的存在，向金属锂负极扩散并与其发生反应，生成 $\mathrm{Li}_{2} \mathrm{~S}_{2} 、 \mathrm{Li}_{2} \mathrm{~S}$ 以及链段长度相对较短的聚硫锂. $\mathrm{Li}_{2} \mathrm{~S}_{2}$ 和 $\mathrm{Li}_{2} \mathrm{~S}$ 会进一步与后续扩散到负极表面的长链聚硫锂发 生反应, 生成链段长度相对较短的聚硫锂, 这些短链聚 硫锂会再次扩散回硫正极, 被氧化成长链聚硫锂, 聚硫 锂在电池正负极间的迁移现象, 被称为飞梭效应 ${ }^{[7 \sim 10]}$.

$$
\begin{aligned}
& 2 \mathrm{Li}+\mathrm{Li}_{2} \mathrm{~S}_{n} \rightarrow \mathrm{Li}_{2} \mathrm{~S} \downarrow+\mathrm{Li}_{2} \mathrm{~S}_{(n-1)} \\
& 2 \mathrm{Li}+\mathrm{Li}_{2} \mathrm{~S}_{n} \rightarrow \mathrm{Li}_{2} \mathrm{~S}_{2} \downarrow+\mathrm{Li}_{2} \mathrm{~S}_{(n-2)} \\
& \mathrm{Li}_{2} \mathrm{~S}+\mathrm{Li}_{2} \mathrm{~S}_{n} \rightarrow \mathrm{Li}_{2} \mathrm{~S}_{k}+\mathrm{Li}_{2} \mathrm{~S}_{(n-k+1)} \\
& \mathrm{Li}_{2} \mathrm{~S}_{2}+\mathrm{Li}_{2} \mathrm{~S}_{n} \rightarrow \mathrm{Li}_{2} \mathrm{~S}_{k}+\mathrm{Li}_{2} \mathrm{~S}_{(n-k+2)}
\end{aligned}
$$

(15) （18)这些化学反应始终存在于 Li-S 电池的体 系中, 但在充电过程中尤为显著, 因为充电过程主要对 应于不溶的 $\mathrm{Li}_{2} \mathrm{~S}_{2}$ 和 $\mathrm{Li}_{2} \mathrm{~S}$ 向易溶于电解液的长链聚硫锂 的转化过程. 而长链聚硫锂向单质硫的转化动力学十分 缓慢, 在首次循环之后硫活性物质主要以 $\mathrm{S}_{n}^{2-}(4 \leqslant n \leqslant 8)$ 大量存在于电解液中 ${ }^{[5]}$, 只有少量活性物质被氧化成 $\mathrm{S}_{8}{ }^{[7]}$. 随着充电过程的进行, 正负极间聚硫离子浓度梯 度不断增加, 聚硫锂向负极扩散动力不断增大, 而其在 负极表面的还原反应也加快. 所以, 充电曲线对应的高
电压平台是聚硫离子电化学氧化和化学还原反应的竞 争过程 ${ }^{[9]}$. 飞梭效应的存在对 Li-S 电池有正反两方面的 影响, 一方面导致电池的自放电, 放电容量低, 以及锂 负极的侵蚀; 另一方面也对电池的过充有一定的保护作 用.

\section{3 硫正极容量损失及衰减机理}

虽然 Li-S 电池活性物质放电比容量高达 1680 $\mathrm{mAh} / \mathrm{g}$, 理论能量密度高达 $2500 \mathrm{Wh} / \mathrm{kg}$, 但目前可实现 能量密度远低于理论值. 电池容量衰减快, 循环寿命短 等问题减慢了 $\mathrm{Li}-\mathrm{S}$ 电池实用化的步伐. 只有分析清楚正 极放电比容量是由哪些部分组成，以及循环过程中活性 物质的转化影响了放电比容量的哪个组成部分, 我们才 能更加深刻地认识引起容量损失的原因, 以及这些原因 是如何导致电池容量衰减的.

根据转移电子数, 按照公式(19) ${ }^{[11]}$, 计算 Li-S 电池 不同放电阶段的放电比容量，结果见表 1 .

$$
q=\frac{n F}{M}
$$

其中 $q$ 为放电比容量, $\mathrm{mAh} / \mathrm{g} ; n$ 为每摩尔单质硫转移电 子数, $\mathrm{mol}^{-1} ; F$ 代表 1 法拉第电量, 为常数值 $26.8 \mathrm{Ah} ; M$ 为单质硫的摩尔质量, $32 \mathrm{~g} / \mathrm{mol}$.

表 1 不同放电深度对应的硫放电比容量

Table 1 The relationship between DOD and the discharge specific capacity

\begin{tabular}{cccc}
\hline 放电产物 & $\begin{array}{c}n \text { 转移电子数 } \\
\left(\mathrm{mol}^{\circ} \cdot \mathrm{mol}^{-1} \mathrm{~S}\right)\end{array}$ & $\begin{array}{c}\text { 放电深度 } \\
\mathrm{DOD}\end{array}$ & $\begin{array}{c}q \text { 放电比容量 } \\
\left(\mathrm{mAh} \cdot \mathrm{g}^{-1}\right)\end{array}$ \\
\hline $\mathrm{S}_{8} \rightarrow \mathrm{S}_{8}^{2-}$ & 0.25 & $12.5 \%$ & 210 \\
$\mathrm{~S}_{8} \rightarrow \mathrm{S}_{6}^{2-}$ & 0.33 & $16.7 \%$ & 280 \\
$\mathrm{~S}_{8} \rightarrow \mathrm{S}_{4}^{2-}$ & 0.5 & $25.0 \%$ & 420 \\
$\mathrm{~S}_{8} \rightarrow \mathrm{Li}_{2} \mathrm{~S}_{2}$ & 1 & $50.0 \%$ & 840 \\
$\mathrm{~S}_{8} \rightarrow \mathrm{Li}_{2} \mathrm{~S}$ & 2 & $100.0 \%$ & 1680 \\
\hline
\end{tabular}

首次实际放电比容量可按如下计算公式归纳为:

$$
\begin{aligned}
& q_{r 1}=\sum \omega_{n} q_{\mathrm{S}_{8} \rightarrow \mathrm{Li}_{2} \mathrm{~S}_{n}} \\
& q_{r 1}=\omega_{8} q_{\mathrm{S}_{8} \rightarrow \mathrm{S}_{8}^{2-}}+\omega_{6} q_{\mathrm{S}_{8} \rightarrow \mathrm{S}_{6}^{2-}}+\omega_{4} q_{\mathrm{S}_{8} \rightarrow \mathrm{S}_{4}^{2-}}+ \\
& \omega_{2} q_{\mathrm{S}_{8} \rightarrow \mathrm{Li}_{2} \mathrm{~S}_{2}}+\omega_{1} q_{\mathrm{S}_{8} \rightarrow \mathrm{Li}_{2} \mathrm{~S}} \\
& q_{r 1}=\omega_{8} \cdot 210+\omega_{6} \cdot 260+\omega_{4} \cdot 420+ \\
& \omega_{2} \cdot 840+\omega_{1} \cdot 1680
\end{aligned}
$$

其中, $q_{r 1}$ 为首次实际放电比容量, $\mathrm{mAh} / \mathrm{g} ; \omega_{n}$ 为转化为 $\mathrm{S}_{n}^{2-}$ 硫量与正极中总硫量的比, $\sum \omega_{n}=1$.

由于放电中间产物聚硫锂 $\mathrm{Li}_{2} \mathrm{~S}_{n}(4 \leqslant n \leqslant 8)$ 易溶于电 解液，在放电循环结束时，部分活性物质以可溶高价态 聚硫锂的形式残留在电解液中，或者是转化为 $\mathrm{Li}_{2} \mathrm{~S}_{2}$ 后， 由于正极结构的导电性变差, 难以进一步转化为放电终 产物 $\mathrm{Li}_{2} \mathrm{~S}$, 这将会导致放电容量低于理论容量. 
在充电循环过程中, 由于将 $\mathrm{S}_{n}^{2-}(4 \leqslant n \leqslant 8)$ 氧化为单 质硫的热力学反应速度十分缓慢, 所以只有少量的活性 物质在充电结束时能够被氧化为单质硫 ${ }^{[7]}$, 大量活性物 质是以 $\mathrm{Li}_{2} \mathrm{~S}_{n}(4 \leqslant n \leqslant 8)$ 的形式存在于电解液中 ${ }^{[3,5]}$. 那么 从第二次放电循环开始, 起始的放电活性物质和首次放 电差别较大，只有少量的活性物质是从单质硫开始放电 反应，而大量的活性物质是从 $\mathrm{Li}_{2} \mathrm{~S}_{n}(4 \leqslant n \leqslant 8)$ 开始放电 反应的. 同时, 由于放电产物 $\mathrm{Li}_{2} \mathrm{~S}_{2}$ 和 $\mathrm{Li}_{2} \mathrm{~S}$ 不易溶于电 解液, 它们沉积在正极中, 会使正极结构的传导性变差, 部分 $\mathrm{Li}_{2} \mathrm{~S}_{2}$ 和 $\mathrm{Li}_{2} \mathrm{~S}$ 会与导电相分离, 从而失去活性, 这 部分中的硫将会造成容量的不可逆损失. 从第二次放电 开始, 实际的放电比容量可归纳为如下计算公式:

$$
\begin{aligned}
& q_{r}^{\prime}=\sum \omega_{m}^{\mathrm{S}_{8}} q_{S_{8} \rightarrow \mathrm{Li}_{2} S_{n}}+\sum \omega_{m}^{\mathrm{S}_{8}^{2-}} q_{\mathrm{S}_{8}^{2-} \rightarrow \mathrm{Li}_{2} \mathrm{~S}_{n}}+ \\
& \sum \omega_{m}^{\mathrm{S}_{6}^{2-}} q_{\mathrm{S}_{6}^{2-} \rightarrow \mathrm{Li}_{2} \mathrm{~S}_{n}}+\sum \omega_{m}^{\mathrm{S}_{4}^{2-}} q_{\mathrm{S}_{4}^{2-} \rightarrow \mathrm{Li}_{2} \mathrm{~S}_{n}}
\end{aligned}
$$

其中

$$
\begin{aligned}
\sum \omega_{m}^{\mathrm{S}_{8}}+\sum \omega_{m}^{\mathrm{S}_{8}^{2-}}+\sum \omega_{m}^{\mathrm{S}_{6}^{2-}}+\sum \omega_{m}^{\mathrm{S}_{4}^{2-}}=1-\frac{\sum \Delta S_{\left(\mathrm{Li}_{2} \mathrm{~S}_{2}+\mathrm{Li}_{2} \mathrm{~S}\right)}}{S} \\
\quad \sum \omega_{m}^{\mathrm{S}_{8}^{2-}} q_{\mathrm{S}_{8}^{2-} \rightarrow \mathrm{Li}_{2} \mathrm{~S}_{n}}=\omega_{6}^{\mathrm{S}_{8}^{2-}} q_{\mathrm{S}_{8}^{2-} \rightarrow \mathrm{S}_{6}^{2-}}+\omega_{4}^{\mathrm{S}_{8}^{2-}} q_{\mathrm{S}_{8}^{2-} \rightarrow \mathrm{S}_{4}^{2-}}+ \\
\quad \omega_{2}^{\mathrm{S}_{8}^{2-}} q_{\mathrm{S}_{8}^{2-} \rightarrow \mathrm{Li}_{2} \mathrm{~S}_{2}}+\omega_{1}^{\mathrm{S}_{8}^{2-}} q_{\mathrm{S}_{8}^{2-} \rightarrow \mathrm{Li}_{2} \mathrm{~S}} \\
=\omega_{6}^{\mathrm{S}_{8}^{2-}} \cdot 70+\omega_{4}^{\mathrm{S}_{8}^{2-}} \cdot 210+\omega_{2}^{\mathrm{S}_{8}^{2-}} \cdot 630+\omega_{1}^{\mathrm{S}_{8}^{2-}} \cdot 1270
\end{aligned}
$$

其余各项以此类推.

总之, 无论何种原因使得活性物质在放电过程中未 能完全转化为 $\mathrm{Li}_{2} \mathrm{~S}$, 或者在充电过程中未能完全转化为 单质硫，结果终将导致放电容量的损失及衰减.

目前, 由正极活性物质导致 Li-S 电池容量衰减的原 因，主要有以下几个方面:

第一，由于活性物质单质硫和固态放电产物 $\mathrm{Li}_{2} \mathrm{~S}_{2}$ 和 $\mathrm{Li}_{2} \mathrm{~S}$ 的绝缘性, 使得单质硫必须与电子导体相复合, 制备成导电剂/硫复合结构, 来增加正极对电子和离子 的传导性. 而导电剂不参与电极反应, 所以降低了正极 的容量 ${ }^{[1 \sim 4]}$.

第二，正极结构破坏. 由于放电中间产物聚硫锂易 溶于电解液, 在充放电过程中, 会从正极结构中溶出, 而放电终产物不溶于电解液, 在电池充放电过程中将会 发生一系列沉淀/溶解反应, 正极活性物质将会在液相 和固相间发生相的转移 ${ }^{[6]}$, 正极结构也会不断的收缩和 膨胀, 这将导致正极结构的失效 ${ }^{[12]}$. 同时, Cheon 等 ${ }^{[9,13 \sim 15]}$ 认为固态放电产物 $\mathrm{Li}_{2} \mathrm{~S}_{2}$ 和 $\mathrm{Li}_{2} \mathrm{~S}$ 在正极上的不均匀沉积, 会使正极导电性变差, 部分 $\mathrm{Li}_{2} \mathrm{~S}_{2}$ 和 $\mathrm{Li}_{2} \mathrm{~S}$ 将会与导电相 分离, 失去活性, 从而导致容量损失及正极结构的破坏. 国防科学技术大学 Diao 等 ${ }^{[16]}$ 研究人员认为, 由于电解 液体系中长链聚硫锂的存在, 它们与 $\mathrm{Li}_{2} \mathrm{~S}_{2}$ 和 $\mathrm{Li}_{2} \mathrm{~S}$ 发生 如方程式(17)和(18)的反应, 提高了 $\mathrm{Li}_{2} \mathrm{~S}_{2}$ 和 $\mathrm{Li}_{2} \mathrm{~S}$ 的可逆 性, 不可逆 $\mathrm{Li}_{2} \mathrm{~S}_{2}$ 和 $\mathrm{Li}_{2} \mathrm{~S}$ 的量在 20 次循环后不超过 $10 \%$.
但是在放电过程中 $\mathrm{Li}_{2} \mathrm{~S}_{2}$ 和 $\mathrm{Li}_{2} \mathrm{~S}$ 将会与电解液分解产物 $\mathrm{LiOR} 、 \mathrm{HCO}_{2} \mathrm{Li}$ 等发生共沉积, 这些电解液分解产物没 有电化学活性, 随着循环的进行, 它们在正极上不断累 积，使得正极导电性变差，从而导致结构的破坏.

第三, 聚硫锂的溶解. 聚硫锂溶解于电解液中, 在 循环终止时, 未能完全转化为终产物, 将会导致一定的 容量损失，但这并不是循环过程中容量衰减的主要原 因 $^{[5]}$. 聚硫锂首次循环后, 在固相和液相间的转移会达 到一定的平衡，电解液中聚硫锂的总含量会保持在相对 稳定值, 并不随着循环的增加而增加, 所以聚硫锂在电 解液中的溶解损失对容量衰减的贡献不大. 但是聚硫锂 的溶解会引起更加严重的问题——飞梭效应”，穿过 隔膜的聚硫锂与金属锂发生如方程(15)和(16)所示的反 应，在负极生成 $\mathrm{Li}_{2} \mathrm{~S}_{2}$ 和 $\mathrm{Li}_{2} \mathrm{~S}$ 针化层，一方面消耗了正 极活性物质，另一方面导致负极的腐蚀及钝化，同时也 会降低电池的库仑效率 ${ }^{[1 \sim 4,17,18]}$.

第四，活性物质不可逆氧化. 国防科技大学 Diao 等 ${ }^{[16]}$ 通过对正极产物的分析，首次发现了 $\mathrm{Li}_{x} \mathrm{SO}_{y}$ 在正极的生 成, 并且沉积量随着循环次数的增加而增多, 这意味着 活性物质的不可逆损失. 由于在循环伏安测试中，很难 区分聚硫锂的逐级氧化峰和长链聚硫锂到 $\mathrm{Li}_{x} \mathrm{SO}_{y}$ 的氧 化峰, 这主要是因为长链聚硫锂被氧化成 $\mathrm{Li}_{x} \mathrm{SO}_{y}$ 是一个 动力学十分缓慢的过程，所以在通常状态下，这个反应 并不明显, 因此始终没有引起研究人员的注意. 但是在 飞梭反应十分活跃的情况下，电池的充电过程被延长， 使得氧化产物 $\mathrm{Li}_{x} \mathrm{SO}_{y}$ 的生成量显著增加，从而为长链聚 硫锂转化为 $\mathrm{Li}_{x} \mathrm{SO}_{y}$ 氧化反应的存在提供了充分的证据. 因此，活性物质的不可逆氧化是导致 Li-S 电池容量衰减 的重要原因之一.

\section{4 提高硫正极性能的研究概述}

目前, 对于提高 Li-S 电池正极性能的研究方向主要 致力于控制活性物质硫的分散和抑制聚硫锂在电解液 中的溶解. 通过采用多孔结构的导电骨架、聚合物包覆、 添加纳米吸收剂等方法来提高硫在正极中的分散性以 及降低聚硫锂的溶解.

\section{1 碳导电结构}

碳材料作为电子的良导体而在电池工业中被广泛 使用, 由于 $\mathrm{Li}-\mathrm{S}$ 电池正极活性物质单质硫的绝缘性, 决 定了碳材料在 Li-S 电池正极中的重要地位. 虽然提高碳 的用量可以增加电极的导电性, 却是以牺牲电池的能量 密度为代价, 所以人们致力于将低密度、高比表面积、 高孔容积并且具有丰富纳米孔道结构的新型碳材料应 用于 $\mathrm{Li}-\mathrm{S}$ 电池正极结构中.

碳纳米管 $(\mathrm{CNT})$ 由于其特殊的结构性能，良好的导 电性、优异的热及机械性能被人们广泛应用于 $\mathrm{Li}-\mathrm{S}$ 电池 正极结构中，构建三维导电网络，改善正极的导电性， 并对硫进行束缚 ${ }^{[19 ~ 28]}$. Ahn 等 ${ }^{[19]}$ 采用化学气相沉积法制 
备了多壁碳纳米管(MWCNTs), 将 MWCNTs 作为导电 剂添加到正极中, 但是首次放电比容量仅有 $485 \mathrm{mAh} / \mathrm{g}$. Qiu 等 ${ }^{[20]}$ 采用类似的方法制备硫包覆 MWCNTs, 壳-核 结构正极材料, 60 次循环后容量保持在 $670 \mathrm{mAh} / \mathrm{g}$. Guo 等 ${ }^{[21]}$ 采用阳极氧化铝膜 (AAO) 作为模板, 制备无序碳纳 米管(DCNTs), 见图 3, 单质硫通过挥发法扩散到 DCNTs 内部结构中, 甚至可以到达管壁的石墨层中, 在 $500{ }^{\circ} \mathrm{C}$ 下制备的 DCNTs $/ \mathrm{S}$ 复合结构正极, 100 次循环后 容量保持率可达 $72.9 \%$.

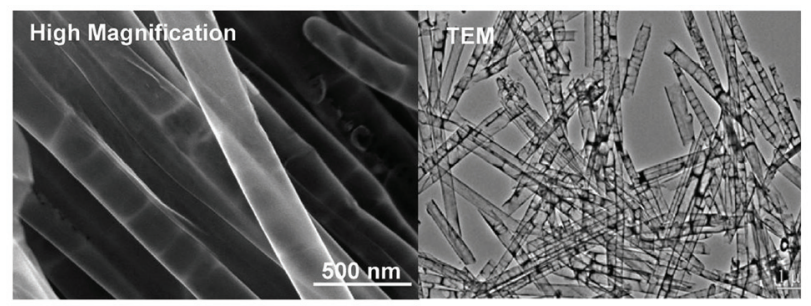

图 3 无序碳纳米管 DCNTs 的 SEM 图和 TEM 图 ${ }^{[21]}$

Figure 3 SEM and TEM images of DCNTs prepared by the template wetting method ${ }^{[21]}$

与碳纳米管结构有着相似之处碳纳米线 (CNF)也受 到 $\mathrm{Li}-\mathrm{S}$ 研究人员的关注. Kim 等 ${ }^{[29]}$ 采用 $\mathrm{CNF}$ 作为导电 添加剂, 首次放电比容量可达 $1191 \mathrm{mAh} / \mathrm{g}, 40$ 次循环后, 容量保持在约 $500 \mathrm{mAh} / \mathrm{g}$. Zhang 等 ${ }^{[30]}$ 采用静电纺丝法 制备碳纤维, 碳化后获得具有多孔结构的碳纤维, 通过 在溶液中进行化学反应将硫沉积在碳纤维的空隙中, 制 备成多孔碳纤维/硫复合结构正极材料, 其原理示意图 见图 4, 在 $0.1 \mathrm{C}$ 倍率下 20 次循环后, 容量可达 1057 $\mathrm{mAh} / \mathrm{g}$. Cui 研究小组 ${ }^{[31]}$ 采用 AAO 为模板, 聚苯乙烯碳 化后沉积在 AAO 壁上, 再将单质硫熔融后渗入 $\mathrm{AAO} /$ 碳结构中, 最后将 AAO 模板去除, 得到空心碳纤维包 覆硫复合正极结构, 原理示意图见图 5, 0.2 C 倍率下 150 次循环后，可逆容量仍保持在 $730 \mathrm{mAh} / \mathrm{g}$.

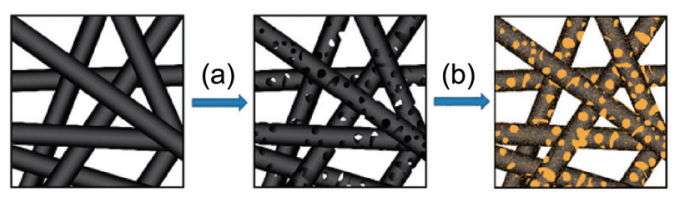

图 4 多孔碳纤维/硫复合正极结构原理示意图 ${ }^{[30]}$

Figure 4 Schematic illustration of the preparation procedures of porous $\mathrm{CNF} / \mathrm{S}$ composite ${ }^{[30]}$
近年来，以 CMK-3 为代表的介孔碳材料，制备的 $\mathrm{C} / \mathrm{S}$ 复合结构正极，引起了研究者们将结构新颖、性能 优异的新型碳材料广泛应用于 Li-S 电池的热潮 ${ }^{[32 ~ 39]}$. 采用熔融法, 在 $155{ }^{\circ} \mathrm{C}$ 单质硫通过毛细力被吸入碳材料 的孔道中, 液态硫在冷却后收缩附着于碳结构的内壁 上. 通过碳硫比的设计可以在结构中预留离子迁移通 道, 以及循环过程中产物体积膨胀所需的空间. 由于丰 富的孔道结构对单质硫和聚硫锂具有一定的吸附作用. 研究人员认为这种 $\mathrm{C} / \mathrm{S}$ 复合结构, 可以提高单质硫在正 极结构中的分散性，限制聚硫离子从正极结构中溶出, 提高电极反应的转化效率, 从而改善正极的性能. Nazar 研究小组 ${ }^{[32]}$ 通过纳米浇注法制备了平均孔径为 3 4 nm 的 CMK-3 型有序阵列介孔碳. 首先采用水热法合成硅 基介孔分子篮 SBA-15, 再以 SBA-15 为模板, 以庶糖为 碳前驱体, 在酸性条件下灌入 SBA-15 孔道中, 形成纳 米有机物/硅复合材料, 然后经过高温炭化, 再用 $\mathrm{HF}$ 或 $\mathrm{NaOH}$ 将模板清洗掉, 最终获得孔道高度有序排列的介 孔碳材料 CMK-3. 将 CMK-3 与单质硫采用熔融法复合 制备 CMK-3/S 复合结构，原理示意图见图 6. 将 $\mathrm{CMK}-3 / \mathrm{S}$ 复合材料采用聚合物包覆后, 首次放电容量 可达 $1320 \mathrm{mAh} / \mathrm{g}, 20$ 次循环后放电容量仍保持在 1100 $\mathrm{mAh} / \mathrm{g}$. Sun 等 ${ }^{[35]}$ 采用与 CMK-3 类似的方法制备了双重 孔道结构有序介孔碳 $(5.6 \mathrm{~nm} / 2.3 \mathrm{~nm})$ 材料, 其首次放电 容量为 $1138 \mathrm{mAh} / \mathrm{g}$, 并在 $6 \mathrm{C}$ 倍率放电下, 显示了良好 的循环性能, 400 次循环后容量仍保持在约 $300 \mathrm{mAh} / \mathrm{g}$. Nazar 研究小组 ${ }^{[40]}$ 也制备了一种具有双重孔道结构的新 型介孔碳 BMC-1 $(5.6 \mathrm{~nm} / 2.0 \mathrm{~nm})$, 采用该介孔碳制备的 $\mathrm{BMC}-1 / \mathrm{S}$ 复合结构正极表现了较好的倍率性能, $1 \mathrm{C}$ 倍 率下首次放电比容量为 $995 \mathrm{mAh} / \mathrm{g}, 100$ 次循环后容量仍 保持在 $550 \mathrm{mAh} / \mathrm{g}$.

Archer 等 ${ }^{[1,42]}$ 制备了一种结构更加新颖的空心碳胶 囊/硫正极结构，首先在高温下裂解先驱体，将碳沉积在 多孔金属或非金属氧化物纳米球表面积内部，再反洗除 去氧化物球, 得到具有图 7(a)所示外壁含有介孔而内部 为空心结构的二维碳球. 最后将空心碳球暴露于硫蒸汽 中, 获得硫负载率达 $70 \%$ 的碳胶囊/硫正极材料, 在 0.5 C 倍率下 100 次循环后可逆容量达 $850 \mathrm{mAh} / \mathrm{g}$.

Dudney 等 ${ }^{[43]}$ 首先采用软模板法制备了孔径为 7.3 $\mathrm{nm}$ 的介孔碳(MPC), 然后用 $\mathrm{KOH}$ 对其进行活化, 在

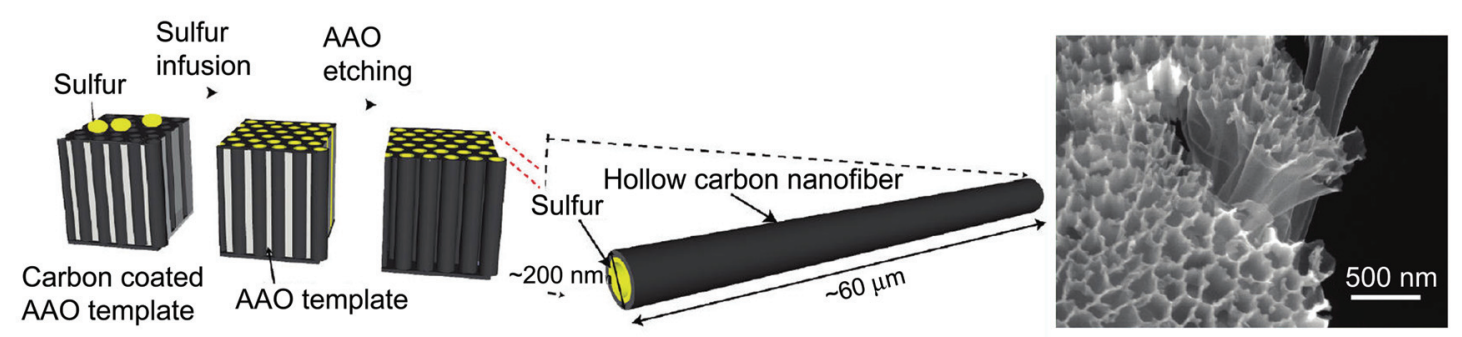

图 5 空心碳纤维包覆硫复合正极结构原理示意图 ${ }^{[31]}$

Figure 5 Schematic of design and fabrication process of hollow carbon nanofibers/sulfur composite structure ${ }^{[31]}$ 

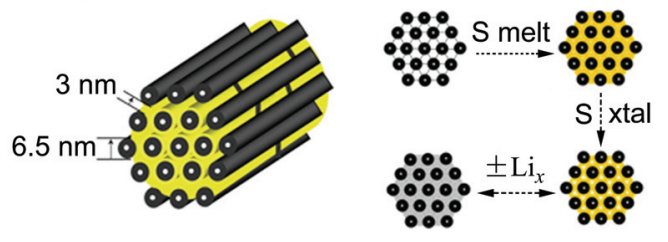

图 $6 \mathrm{CMK}-3 / \mathrm{S}$ 复合正极结构原理示意图 ${ }^{[32]}$

Figure 6 A schematic diagram of the sulfur confined in the interconnected pore structure of CMK- $3^{[32]}$
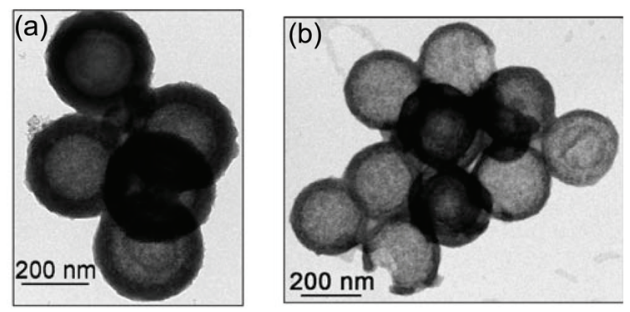

图 7 空心碳球 $(\mathrm{a})$ 和空心碳胶囊/硫正极结构(b $)^{[4]}$

Figure 7 TEM images of (a) mesoporous carbon hollow spheres and (b) C@S nanocomposite ${ }^{[4]}$

MPC 的孔壁上生成孔径小于 $2 \mathrm{~nm}$ 的微孔, 最后获得具 有双重孔道结构的碳材料 a-MPC. 微孔用于负载单质 硫, 并为其传导电子提供有效的路径, 介孔用于传输 $\mathrm{Li}^{+}$ 及容纳循环过程中产生的聚硫锂, 其原理示意图见图 8. 采用双重孔道 $\mathrm{a}-\mathrm{MPC} / \mathrm{S}$ 复合正极电池首次放电比容量 高达 $1584 \mathrm{mAh} / \mathrm{g}, 50$ 次循环后容量仍保持在 $805 \mathrm{mAh} / \mathrm{g}$.

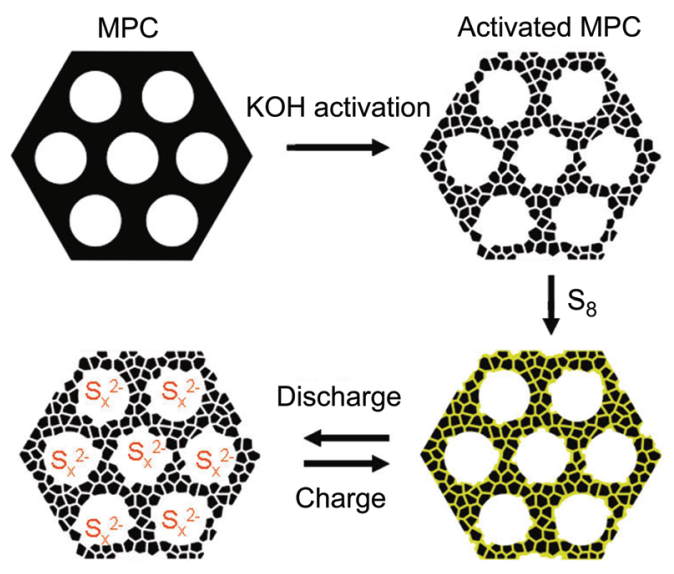

图 8 双重孔道 a-MPC/S 复合正极结构原理示意图 ${ }^{[43]}$

Figure 8 Illustration of a-MPC/S composite cathode material by using a bimodal porous carbon $^{[43]}$

Gao 等 ${ }^{[4]}$ 采用蔗糖作为碳源制备了内部具有孔径 小于 $1 \mathrm{~nm}$ 微孔结构的碳球, 并将硫熔融渗入碳球内部, 制备 $\mathrm{S}$ /碳球复合正极材料, 原理示意图见图 9. 该结构 表现出优异的循环性能, 500 次循环后可逆容量仍保持 在 $650 \mathrm{mAh} / \mathrm{g}$.

石墨烯由于其高导电性、高比表面积、易表面改性, 而在 Li-S 电池中备受瞩目 ${ }^{[45 ~ 59]}$. Liu 等 ${ }^{[45]}$ 将硫颗粒填充 在石墨烯片层间, 制备 “三明治” 结构石墨烯/硫(FGSS) 纳米复合结构, 利用离子交换型 Nafion 膜对 FGSS 材料
进行包覆，制备的正极材料在 $1 \mathrm{C}$ 倍率下, 100 次循环后 可逆放电容量可达 $505 \mathrm{mAh} / \mathrm{g}$. Dai 等 ${ }^{[48]}$ 首先将聚乙二醇 (PEG)包覆在硫颗粒上，再将用碳黑颗粒修饰的氧化石 墨烯 $(\mathrm{GO})$ 组装在硫颗粒外部, 制备成氧化石墨烯/硫复 合结构正极材料, 其原理示意图见图 10, 100 次循环后, 可逆容量保持在 $600 \mathrm{mAh} / \mathrm{g}$. Zhang 等 ${ }^{[60]}$ 采用氧化石墨 热膨胀法制备 HPG-1000 型石墨烯, 再将熔融硫渗入石 墨烯孔隙中, 得到多孔石墨烯/硫复合结构, 原理结构示 意图见图 11, 在 $10 \mathrm{C}$ 倍率下放电容量可达 $543 \mathrm{mAh} / \mathrm{g}$.

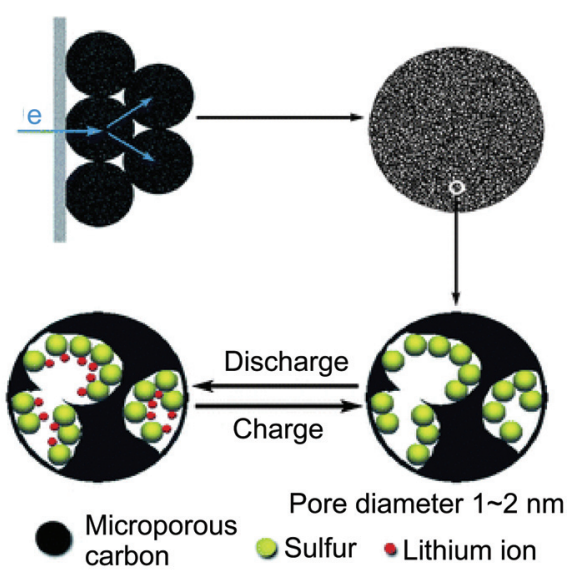

图 9 硫/微孔碳球复合结构原理示意图 ${ }^{[44]}$

Figure 9 A scheme of the constrained sulfur inside the micropores of the sulfur-carbon sphere composite ${ }^{[4]]}$

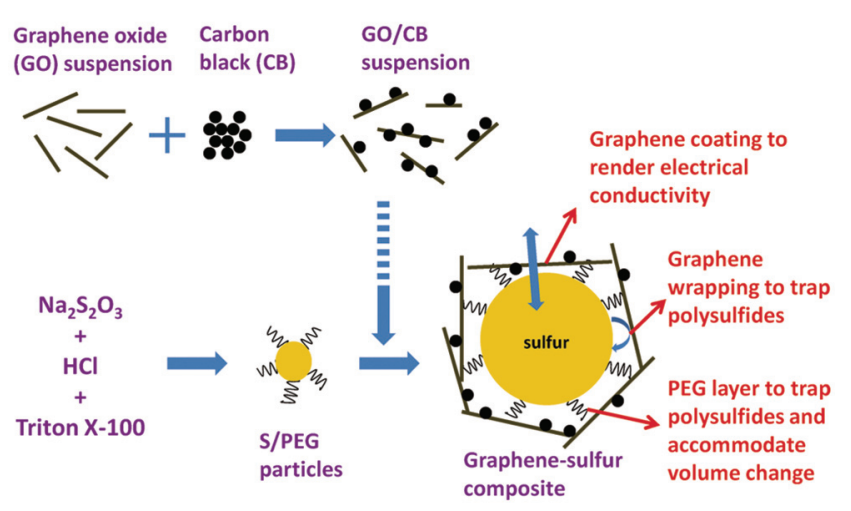

图 10 石墨烯/硫复合结构原理示意图 ${ }^{[48]}$

Figure 10 Schematic of the synthesis steps for a graphene/sulfur composite structure ${ }^{[48]}$

另外, Aurbach 研究小组 ${ }^{[61]}$ 将熔融硫渗入含有微孔 结构的碳纤维布中, 在无需加入粘结剂的情况下, 制备 活性碳纤维布/硫复合正极, 80 次循环后可逆容量仍然 可达 $800 \mathrm{mAh} / \mathrm{g}$.

\section{2 聚合物包覆硫活性物质}

除了碳材料, 有机聚合物材料也在 Li-S 电池中得到 了广泛的应用，它们一方面可以通过导电聚合物来改善 硫正极的导电性，另一方面可以利用有机聚合物包覆硫 或碳/硫复合材料，通过物理阻隔的方法来抑制聚硫锂 在电解液中的溶解损失. 

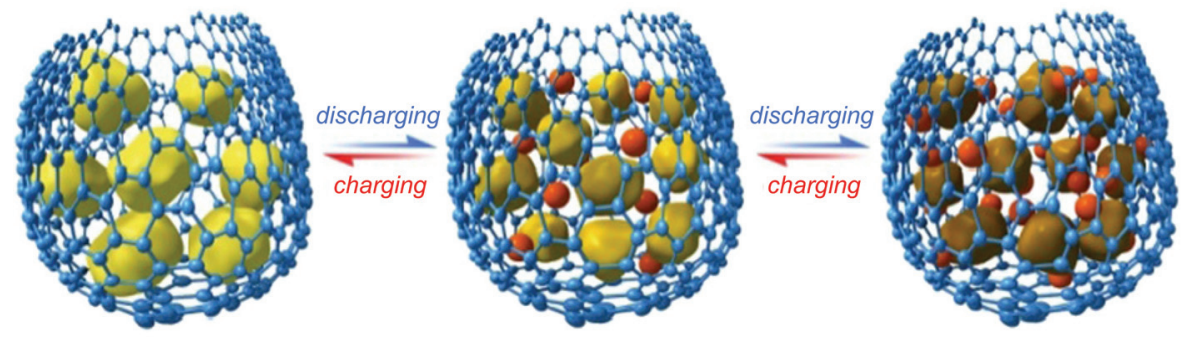

图 11 多孔石墨烯/硫复合结构原理示意图 ${ }^{[60]}$

Figure 11 Schematic illustration of entrapment of sulfur in graphene ${ }^{[60]}$

上海交通大学 Wang 等 ${ }^{[62 ~ 64]}$ 利用聚丙烯腈(PAN)包 覆单质硫来改善正极的导电性, 降低硫的颗粒尺寸, 抑 制聚硫锂的溶解. 将 PAN 颗粒与升华硫, 在 $300{ }^{\circ} \mathrm{C}$ 惰性 气氛下共融 $6 \mathrm{~h}, \mathrm{PAN}$ 经过了脱 $\mathrm{H}_{2} \mathrm{~S}$ 过程后生成主链含有 聚苯烯结构的导电化合物, 制备了分子水平接触的聚丙 烯腈包覆硫复合材料, 采用凝胶电解质在 50 次循环后 容量仍保持在 $600 \mathrm{mAh} / \mathrm{g}$. 该研究小组 ${ }^{[65]}$ 也将丙烯腈在 MWCNT 表面进行原位聚合, 制备PAN@MWCNT 复合 材料, 再将升华硫与 PAN@MWCNT 在低温下热解, 获 得 pPAN-S@MWCNT 复合正极材料, 其合成原理示意 图见图 12. 该材料由内外两种导电材料聚丙烯腈和 MWCNT, 构建了良好的核壳结构导电网络, 显示了良 好的倍率性能, $4 \mathrm{C}$ 倍率下放电容量可达 $550 \mathrm{mAh} / \mathrm{g}$. 同 时, 他们通过原位聚合法制备了聚丙烯腈/石墨烯 (PAN/GNS) 复合材料, 再采用低温热解的方法制备 pPAN-S/GNS 复合正极材料, 其合成原理示意图见图 13. 该材料首次放电容量可达 $1500 \mathrm{mAh} / \mathrm{g}, 6 \mathrm{C}$ 倍率下 放电容量仍可保持在 $800 \mathrm{mAh} / \mathrm{g}^{[66,67]}$.

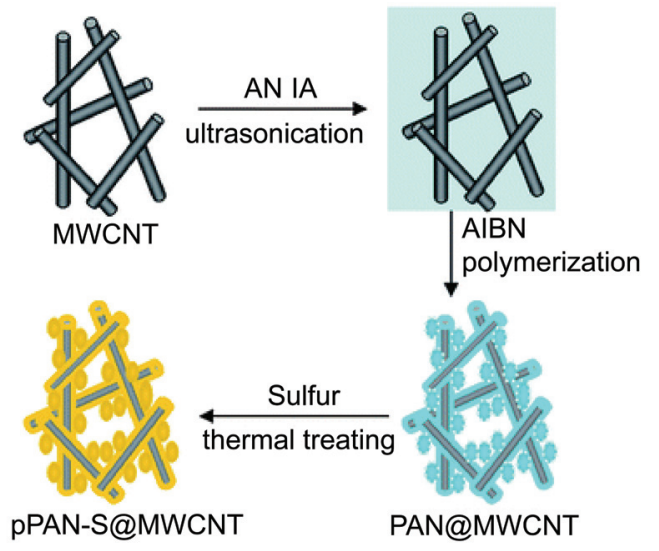

图 12 原位聚合法制备 pPAN-S@MWCNT 复合正极材料原理示意图 ${ }^{[65]}$ Figure 12 Schematic of in situ polymerization and systemization of pPAN-S@MWCNT composite ${ }^{[65]}$

聚吡咯(PPy)作为一种优异的导电聚合物被广泛应 用于 Li-S 电池中 ${ }^{[68 ~ 77]}$. 将 PPy 均匀包覆在硫颗粒表面 上, 不仅可以提高硫的导电性, PPy 也起到了粘结剂的 作用，使得颗粒与颗粒之间紧密结合，提高电子在颗粒
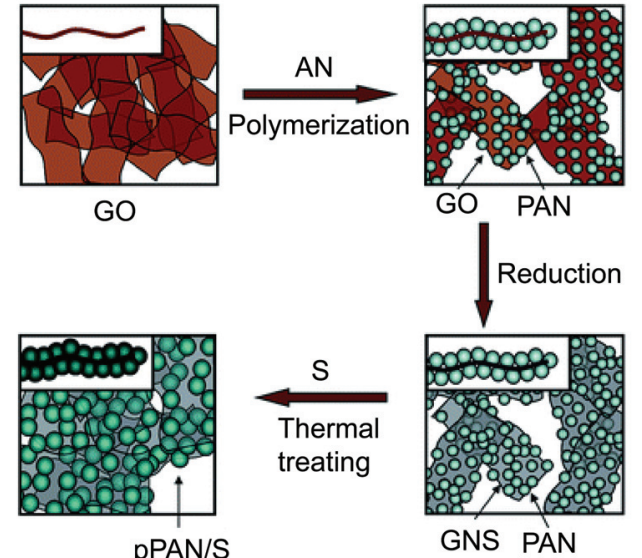

图 13 原位聚合法合成 pPAN-S/GNS 复合正极材料原理示意图 ${ }^{[66,67]}$ Figure 13 Schematic of in situ polymerization and synthesis of the pPAN-S/GNS composite ${ }^{[6,67]}$

之间的传导能力. 同时, PPy 有一定的电化学活性, 有利 于提高正极的放电容量. Wang 等 ${ }^{[68]}$ 利用聚吡咯来改善 硫正极的导电性，采用对甲苯磺酸钠作为掺杂剂，4-苯 乙烯磺酸钠为表面活性剂, $0.1 \mathrm{~mol} / \mathrm{L} \mathrm{FeCl}_{3}$ 作为氧化剂, 通过化学聚合法制备了 PPy, 然后将单质硫与 PPy 共融, 制备 S/PPy 复合材料. 该材料在 20 次循环后容量可达 $800 \mathrm{mAh} / \mathrm{g}$. 北京航空航天大学 Zhang 等 ${ }^{[69]}$ 采用十六烷 基-三甲基溴化铵 (CTAB)作为软模板, 通过化学聚合法 制备了聚吡咯纳米线, 再与单质硫在 $150{ }^{\circ} \mathrm{C}$ 共融, 制备 S/PPy 纳米线复合材料, 20 次循环后容量可保持在 570 $\mathrm{mAh} / \mathrm{g}$. 该研究小组采用类似的方法制备硫/聚(吡咯-苯 胺)(S/PPyA)纳米线复合材料, 首次放电容量可达 1285 $\mathrm{mAh} / \mathrm{g}, 40$ 次循环后容量仍保持在 $866 \mathrm{mAh} / \mathrm{g}^{[70]}$. 中科 院上海陶瓷研究所 Wen 等 ${ }^{[71 ~ 73]}$ 采用模板自分解法制备 了管状聚吡咯纳米线(T-PPy), 在 $150{ }^{\circ} \mathrm{C}$ 下与升华硫共 融, 将硫渗入 T-PPy 的管状结构中, 制备了硫含量为 $30 \%$ 的 T-PPy包覆硫复合结构正极材料, 在 80 次循环后, 容量仍保持在 $650 \mathrm{mAh} / \mathrm{g}$.

北京理工大学 $\mathrm{Wu}$ 等 ${ }^{[78,79]}$ 通过原位化学氧化聚合法 将聚噻吩 (PTh)包覆在硫颗粒表面上, 制备硫含量达 $70 \%$ 的 S/PTh 核壳结构复合材料, 合成原理示意图见图 14. 聚噻吩一方面可以增强正极材料的导电性, 另一方 


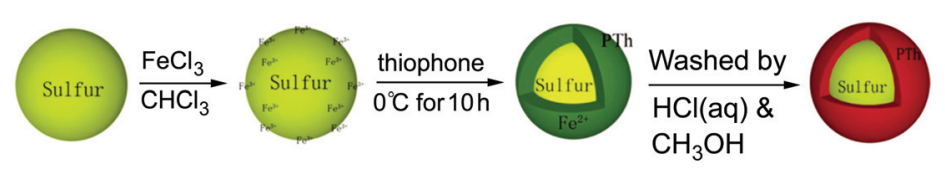

图 14 硫/聚噻吩核壳结构正极材料制备过程原理示意图 [79]

Figure 14 Synthesis procedure of the S/PTh composites ${ }^{[79]}$

面由于其多孔结构可以抑制聚硫锂的溶解. 首次放电容 量可达 $1119 \mathrm{mAh} / \mathrm{g}, 80$ 次循环后容量仍保持在 811 $\mathrm{mAh} / \mathrm{g}$. 该研究小组采用类似的方法将导电聚苯胺 (PANi)包覆在以多壁碳纳米管为核、硫为壳的复合材料 外部, 制备 PANi-S/MWCNT 复合结构正极材料, 首次 放电容量可达 $1334 \mathrm{mAh} / \mathrm{g}, 80$ 次循环后容量仍保持在 $932 \mathrm{mAh} / \mathrm{g}^{[80]}$.

南开大学 $\mathrm{Gao}$ 等 ${ }^{[81]}$ 采用球磨和热处理两步法制备 了导电炭黑和升华硫复合材料, 然后再采用原位化学氧 化聚合法制备聚苯胺包覆硫碳核壳结构复合正极材料 (PANi@S/C), 聚苯胺包覆层厚度约为 5 10 nm, 硫负 载率为 $43.7 \%$ 时, $10 \mathrm{C}$ 倍率下最高放电比容量可达 635 $\mathrm{mAh} / \mathrm{g}, 200$ 次循环后容量保留率仍高于 $60 \%$. 武汉大学 Cao 等 ${ }^{[82]}$ 采用自组装方法制备了聚苯胺纳米管 (PANi-NT) 来对活性物质硫进行束缚, 在 $280{ }^{\circ} \mathrm{C}$ 下与硫 进行热处理, 部分硫将会与 PANi-NT 发生反应, 生成具 有稳定 3D 交联结构的硫-聚苯胺聚合物(SPANi-NT). 该 结构不仅对硫及硫化锂具有较强的物理和化学束缚作 用, 在充放电过程中聚硫锂能够实现原位可逆电化学反 应，其结构原理示意图见图 15. SPAN-NT 正极材料在 $0.1 \mathrm{C}$ 倍率下 100 次循环后容量仍可达 $837 \mathrm{mAh} / \mathrm{g}$, 而在 $1 \mathrm{C}$ 倍率下放电 500 次循环后容量仍可保持在 420 $\mathrm{mAh} / \mathrm{g}$.

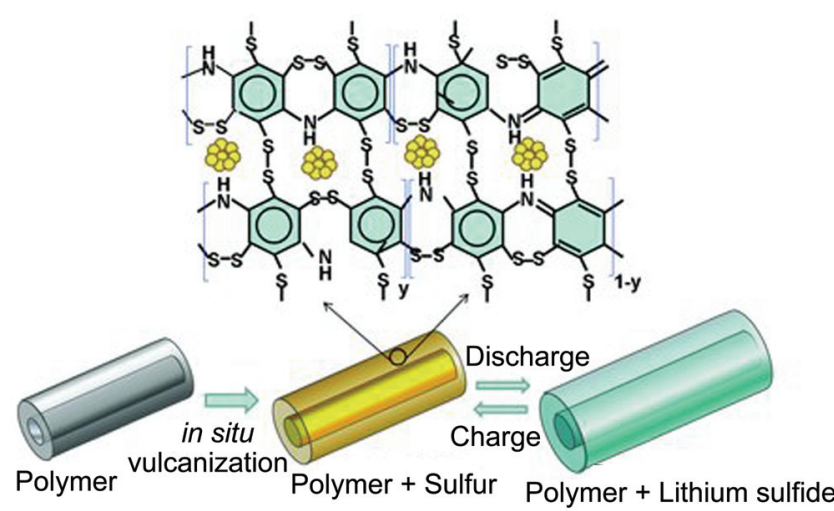

图 15 SPAN-NT 正极材料结构原理示意图 ${ }^{[82]}$

Figure 15 Schematic illustration of the construction of the SPANi-NT composite $^{[82]}$

Cui 等 ${ }^{[83]}$ 为了更加有效地抑制聚硫锂的溶解, 采用 结构稳定且强度适中的聚二氧噻吩-聚苯乙烯磺酸酯 (PEDOT:PSS) 导电聚合物包覆 CMK-3/S 复合材料, 其合 成原理示意图见图 16. 该材料相比于 $\mathrm{CMK}-3 / \mathrm{S}$ 复合正 极材料, 100 次循环放电比容量约有 $10 \%$ 的提高.

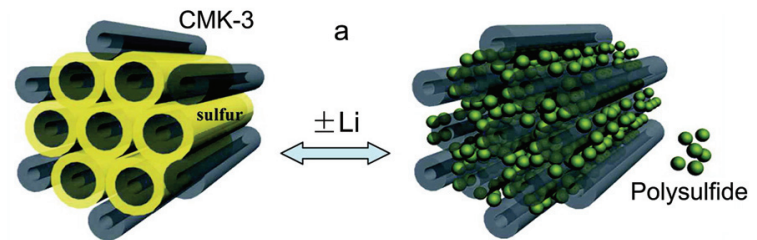

CMK-3/S composite

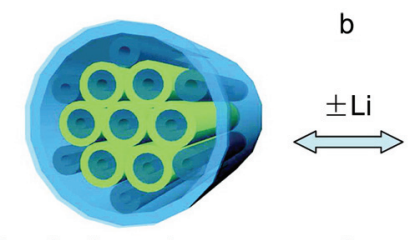

Polysulfide dissolution

Conductive polymer wrapped CMK-3/S composite

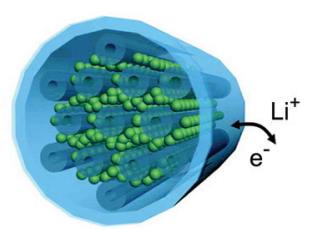

Polysulfide containment

图 16 PEDOT:PSS 包覆 CMK-3/S 复合正极材料原理示意图 ${ }^{[83]}$ Figure 16 Scheme of PEDOT:PSS-coated CMK-3/sulfur composite ${ }^{[83]}$

\section{3 纳米金属氧化物添加剂}

在 Li-S 电池研究初期, 为了抑制聚硫锂向电解液中 的溶解，提高正极活性物质的转化效率，人们将纳米金 属氧化物添加剂加入正极材料中, 如氧化矾、氧化硅、 氧化铝及过渡金属氧化物，以期利用它们的高比表面积 及表面基团, 对聚硫锂产生一定的物理和化学吸附作 用 ${ }^{[84-91]}$.

$\mathrm{Kim}$ 等 ${ }^{[84]}$ 采用溶胶一凝胶法制备了纳米级镍氧化物 $\left(\mathrm{Mg}_{0.6} \mathrm{Ni}_{0.4} \mathrm{O}\right)$ 颗粒, 作为非活性组分加入 $\mathrm{Li}-\mathrm{S}$ 电池正极 材料中, 对抑制聚硫锂向电解液中溶解以及促进氧化还 原反应产生了积极的效果, 改善了材料的倍率性能及循 环性能, 最高放电比容量可达 $1185 \mathrm{mAh} / \mathrm{g}, 50$ 次循环后 容量保留率高于 $85 \%$. Chen 等 ${ }^{[88,89]}$ 采用自蔓延高温合成 法也制备了纳米结构 $\mathrm{Mg}_{0.6} \mathrm{Ni}_{0.4} \mathrm{O}$, 并利用其通过球磨制 备了硫/聚丙烯腈/ $\mathrm{Mg}_{0.6} \mathrm{Ni}_{0.4} \mathrm{O}\left(\mathrm{S} / \mathrm{PAN} / \mathrm{Mg}_{0.6} \mathrm{Ni}_{0.4} \mathrm{O}\right)$ 复合正 极材料, 从第二次循环开始放电比容量可达 1223 $\mathrm{mAh} / \mathrm{g}, 100$ 次循环后容量保持率可达 $100 \%$.

Nazar 研究小组 ${ }^{[87}$ 将不同形态的介孔氧化钛 $\left(\mathrm{TiO}_{2}\right)$ 与孔内含有升华硫的介孔碳材料(孔径大于 $10 \mathrm{~nm}$ ), 按 照 $10 ： 1$ 比例制备复合正极材料, 获得了纳米吸收剂物 理性能与其对聚硫锂吸附能力的关系, 并发现由于聚硫 负离子与氧化物表面静电力的作用, 在充放电过程中聚 硫锂将优先被吸附在 $\mathrm{TiO}_{2}$ 的孔道内. 比表面积最高, 平 均粒径为 $5 \mathrm{~nm}$ 的 $\alpha-\mathrm{TiO}_{2}$ 复合正极表现出最优异的电性 能, 首次放电容量可达 $1201 \mathrm{mAh} / \mathrm{g}, 100$ 次循环后容量 保留率可达 $73 \%$, 在高倍率下 200 次循环后容量仍可保 
持在 $750 \mathrm{mAh} / \mathrm{g}$. 该研究小组采用表面自生长法, 在介 孔碳/硫复合材料表面生成均匀的氧化物层 $\mathrm{MO}_{x}(\mathrm{M}=$ $\mathrm{Si}, \mathrm{V}), \mathrm{MO}_{x}$ 绝缘包覆层可以阻止聚硫锂在正极表面反 应生成不溶的阻挡层, 并且可以限制聚硫锂的溶解, $\mathrm{MO}_{x}$-coated $\mathrm{CMK}-3 / \mathrm{S}$ 复合正极材料合成原理示意图见 图 17. $2.7 \% \mathrm{SiO}_{x}$ 包覆 $\mathrm{CMK}-3 / \mathrm{S}$ 复合材料可逆容量可达 $718 \mathrm{mAh} / \mathrm{g}, 60$ 次循环后容量保留率为 $82.5 \%$. 相比于未 包覆 $\mathrm{MO}_{x}$ 的 $\mathrm{CMK}-3 / \mathrm{S}$ 正极材料, 可逆放电容量有所下 降可能是由于 $\mathrm{MO}_{x}$ 的绝缘性导致的, 但是由于其阻挡 能力强对提高电池的循环性能具有积极的作用 ${ }^{[90]}$.

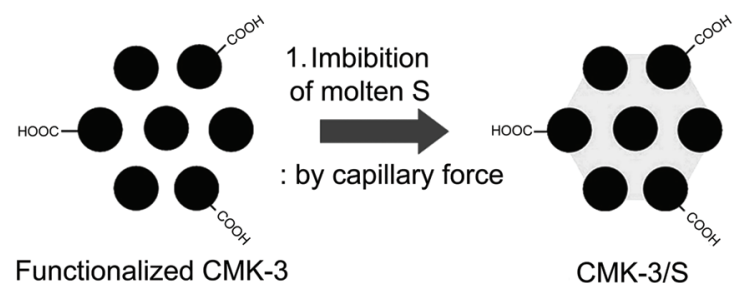

Functionalized CMK-3

CMK-3/S

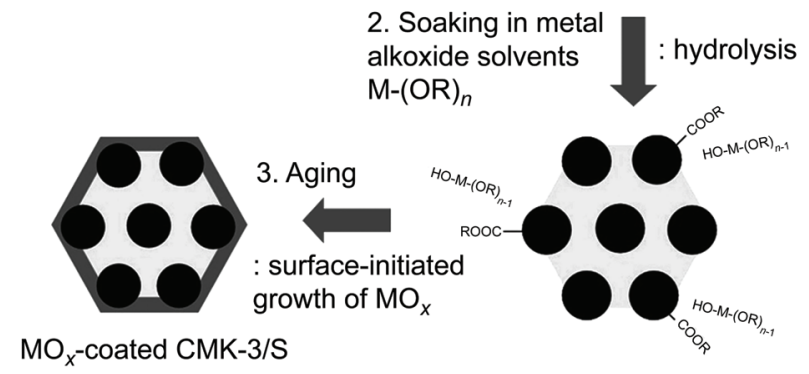

图 $17 \mathrm{MO}_{x}$-coated CMK-3/S 复合正极材料合成原理示意图 ${ }^{[0]}$

Figure 17 Schematic diagram for the synthesis of $\mathrm{MO}_{x}$-coated CMK-3/S composite ${ }^{[90]}$

\section{5 展望}

在过去的几十年中, 通过研究人员的不解努力, Li-S 电池的性能已经获得了显著的提高, 尤其在硫正极 方面采用孔道丰富、结构新颖的碳材料、导电聚合物以 及纳米金属氧化物材料, 在提高正极导电性能、改善正 极结构、抑制聚硫溶解方面取得了长足的进步.

然而, 无论利用碳材料还是导电聚合物材料对硫活 性物质进行束缚, 抑制聚硫锂从正极结构中溶出, 在电 池充放电过程中, 都很难按照这种理想的正极结构模型 来运行, 即使聚硫锂的溶出得到一定程度的缓解, 但是 仍然不能做到完全控制. 在充电过程中, 当脱离了正极 结构的聚硫锂再次向正极内部扩散时, 这种优化设计的 正极结构又会成为物理阻隔屏障, 而反作用于溶出的聚 硫锂. 另外, 导电聚合物/硫复合材料对硫的负载率虽然 可以高达 $70 \%$, 但是该材料的导电性仍然不能满足 Li-S 电池正极的需要, 所以仍然需要加入 $20 \% \sim 30 \%$ 的导电 碳材料, 这样就会降低硫在正极材料中的比例, 不利于 电池能力密度的提高. 而纳米金属氧化物吸收剂, 由于 其本身不具有电化学活性和绝缘性, 对改善电极的导电 性没有积极作用, 反而会降低电池的能量密度.
在上述诸多方法中，性能最佳的硫正极可逆容量保 持在 $1200 \mathrm{mAh} / \mathrm{g}^{[89]}$, 距离硫的理论放电比容量 1680 $\mathrm{mAh} / \mathrm{g}$ 仍然有一定的差距. 所以, 除了优化正极导电结 构, 抑制聚硫锂溶出以外, 其他方面导致硫电极性能恶 化的原因也需要引起研究人员的注意:

(1) 电解液分解产物在正极表面沉积，形成的不溶 有机物阻挡层而引起的正极传导性降低，导致正极结构 失效, 降低硫活性物质的转化效率 ${ }^{[5]}$. 而这并不能仅通 过改善正极结构而得到有效的解决，需要开发适用于 Li-S 电池的新型稳定电解液体系.

(2)硫活性物质的不可逆氧化，导致大量 $\mathrm{Li}_{x} \mathrm{SO}_{y}$ 在 正极的富集，不仅会加速正极结构的破坏，更加意味着 活性物质的不可逆损失 ${ }^{[16]}$. 这就需要一方面从抑制硫 活性物质氧化着手，寻求适当的抗氧化添加剂. 另一方 面从减弱 “飞梭效应” 着手, 降低硫活性物质氧化的机 会.

另外，除了正极方面的问题，电解液随着循环而枯 竭以及金属锂负极的腐蚀, 这些作为 Li-S 电池的重要组 成部分不应该将其割裂而分开对待. 只有将各部分问题 结合起来，综合考虑，才能真正推进 Li-S 电池实用化进 程.

\section{作者简介}

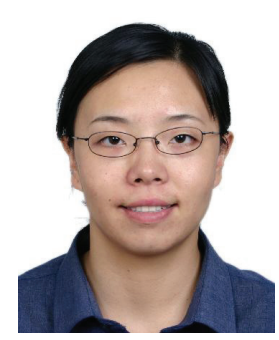

习岩，女，1981 年出生，在读博士，国防科学技术大学航 天科学与工程学院学员. 2004 年在哈尔滨工业大学市政环境 工程学院获得环境工程专业学士学位. 2006 年在国防科学技 术大学航天科学与技术学院获得高分子化学与物理专业硕士 学位. 2007 年至 2008 年在中国人民解放军海军工作. 2009 年 至今在国防科学技术大学航天科学与工程学院攻读博士学位, 师从谢凯教授, 从事关于 Li-S 电池方向研究.

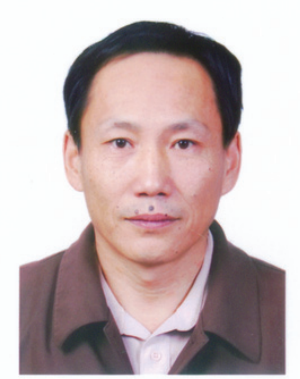

谢凯，博士生导师，教授. 1983 年毕业于浙江大学基本有 机化工专业, 获工学学士学位; 1988 年毕业于国防科技大学应 用化学专业, 获工学硕士学位. 1993 年晋升副教授, 1999 年晋 升教授. 中国材料研究学会理事, 中国空间学会理事, 享受国 
务院特殊津贴的专家. 从事过的专业包括高分子材料、光电功 能材料、纳米材料、能源材料等. 现主要研究领域: 高能量密 度锂离子二次电池及材料、锂硫二次电池及材料、固体薄膜 电池、新型薄膜太阳能电池、光电功能材料等. 在国内外学术 刊物上发表论文 80 余篇, 被 SCI、EI、IAA 检索 40 余篇.

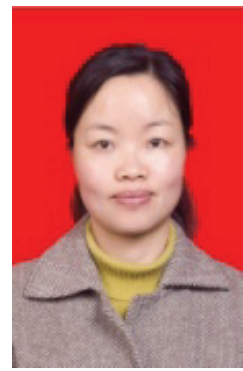

洪晓斌, 国防科学技术大学材料科学与工程系副教授. 1993 年在哈尔滨工业大学应用化学系获得学士学位, 1996 年 在航天科技集团第四研究院获得硕士学位, 2009 年在国防科 学技术大学获得博士学位(在职), 导师谢凯教授. 主要研究领 域为高性能锂硫电池, 锂电池新型聚合物电解质.

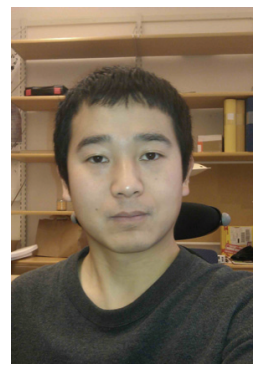

熊仕昭, 国防科学技术大学航天科学与工程学院在读博 士生. 2008 年在国防科学技术大学获得学士学位, 2010 年在国 防科学技术大学获得硕士学位, 主要研究领域为锂负极与锂 硫二次电池.

\section{References}

[1] Bruce, P. G.; Freunberger, S. A.; Hardwick, L. J.; Tarascon, J. M. Nat. Mater. 2012, 11, 19.

[2] Ellis, B. L.; Lee, K. T.; Naza, L. F. Chem. Mater 2010, $22,691$.

[3] Ji, X.; Nazar, L. F. J. Mater. Chem. 2010, 20, 9821.

[4] Evers, S.; Nazar, L. F. Acc. Chem. Res. 2012, DOI: 10.1021/ $\operatorname{ar} 3001348$.

[5] Diao, Y.; Xie, K.; Xiong, S.; Hong, X. J. Electrochem. Soc. 2012, $159, \mathrm{~A} 421$.

[6] Kumaresan, K.; Mikhaylik, Y.; White, R. E. J. Electrochem. Soc. 2008, 155, A576.

[7] Mikhaylik, Y. V.; Akridge, J. R. J. Electrochem. Soc. 2004, 151, A1969.

[8] Kolosnitsyn, V. S.; Karaseva, E. V. Russ. J. Electrochem. 2008, 44, 506.

[9] Cheon, S. E.; Ko, K. S.; Cho, J. H.; Kim, S. W.; Chin, E. Y. J. Electrochem. Soc. 2003, 150, A796.

[10] Akridge, J. R.; Mikhaylik, Y. V.; White, N. Solid State Ionics 2004, $175,243$.

[11] Hamann, C. H.; Hamnett, A.; Vielstich, W. Electrochemistry, 2nd ed., Chemical Industry Press, Beijing, 2009. (卡尔・哈曼, 活尔 夫・菲尔施蒂希, 安德鲁・哈姆内特, 电化学, 第二版, 化学工业 出版社, 北京, 2009.)

[12] He, X.; Ren, J.; Wang, L.; Pu, W.; Jiang, C.; Wan, C. J. Power Sources 2009, 190, 154.

[13] Cheon, S. E.; Ko, K. S.; Cho, J. H.; Kim, S. W.; Chin, E. Y. $J$. Electrochem. Soc. 2003, 150, A800.

[14] Cheon, S. E.; Choi, S. S.; Han, J. S.; Choi, Y. S.; Jung, B. H.; Lima,
H. S. J. Electrochem. Soc. 2004, 151, A2067.

[15] Elazari, R.; Salitra, G.; Talyosef, Y.; Grinblat, J.; Kelley, C. S.; Xiao, A.; Affinito, J.; Aurbach, D. J. Electrochem. Soc. 2010, 157, A1131.

[16] Diao, Y.; Xie, K.; Xiong, S.; Hong, X. J. Electrochem. Soc. 2012, 159, A1816.

[17] Barchasz, C.; Leprêtre, J. C.; Alloin, F.; Patoux, S. J. Power Sources 2011.

[18] Barchasz, C.; Molton, F.; Duboc, C.; Leprêtre, J. C.; Patoux, S.; Alloin, F. Anal. Chem. 2011, 84, 3973.

[19] Han, S. C.; Song, M. S.; Lee, H.; Kim, H. S.; Ahn, H. J.; Lee, J. Y. J. Electrochem. Soc. 2003, 150, A889.

[20] Yuan, L.; Yuan, H.; Qiu, X.; Chen, L.; Zhu, W. J. Power Sources 2009, 189, 1141.

[21] Guo, J.; Xu, Y.; Wang, C. Nano Lett. 2011, 11, 4288.

[22] Wei, W.; Wang, J.; Zhou, L.; Yang, J.; Schumann, B.; NuLi, Y. Electrochem. Commun. 2011, 13, 399.

[23] Ahn, W.; Kim, K. B.; Jung, K. N.; Shin, K. H.; Jin, C. S. J. Power Sources 2012, 202, 394.

[24] Chen, J. J.; Zhang, Q.; Shi, Y. N.; Qin, L. L.; Cao, Y.; Zheng, M. S.; Dong, Q. F. Phys. Chem. Chem. Phys. 2012, 14, 5376.

[25] Dörfler, S.; Hagen, M.; Althues, H.; Tübke, J.; Kaskel, S.; Hoffmann, M. J. Chem. Commun. 2012, 48, 4097.

[26] Su, Y. S.; Fu, Y.; Manthiram, A. Phys. Chem. Chem. Phys. 2012, 14, 14495.

[27] Zhou, G. M.; Wang, D. W.; Li, F.; Hou, P. X.; Yin, L. C.; Liu, C.; Lu, G. Q.; Gentle, I. R.; Cheng, H. M. Energy Environ. Sci. 2012, 5 , 8901 .

[28] Zheng, W.; Liu, Y. W.; Hu, X. G.; Zhang, C. F. Electrochim. Acta 2006, 51,1330 .

[29] Choi, Y. J.; Kim, K. W.; Ahn, H. J.; Ahn, J. H. J. Alloys Compd. 2008, 449, 313.

[30] Ji, L.; Rao, M.; Aloni, S.; Wang, L.; Cairns, E. J.; Zhang, Y. Energy Environ. Sci. 2011, 4, 5053.

[31] Zheng, G.; Yang, Y.; Cha, J. J.; Hong, S. S.; Cui, Y. Nano Lett. 2011, 11,4462 .

[32] Ji, X.; Lee, K. T.; Nazar, L. F. Nat. Mater. 2009, 8, 500.

[33] Wang, J.; Chew, S. Y.; Zhao, Z. W.; Ashraf, S.; Wexler, D.; Chen, J.; Ng, S. H.; Chou, S. L.; Liu, H. K. Carbon 2008, 46, 229.

[34] Lai, C.; Gao, X. P.; Zhang, B.; Yan, T. Y.; Zhou, Z. J. Phys. Chem. C 2009, 113, 4712.

[35] Chen, S. R.; Zhai, Y. P.; Xu, G. L.; Jiang, Y. X.; Zhao, D. Y.; Li, J. T.; Huang, L.; Sun, S. G. Electrochim. Acta 2011, 56, 9549.

[36] Li, X.; Cao, Y.; Qi, W.; Saraf, L. V.; Xiao, J.; Nie, Z.; Mietek, J.; Zhang, J. G.; Schwenzer, B.; Liu, J. J. Mater. Chem. 2011, 21 , 16603.

[37] Liang, X.; Wen, Z.; Liu, Y.; Zhang, H.; Huang, L.; Jin, J. J. Power Sources 2011, 196, 3655.

[38] Schuster, J.; He, G.; Mandlmeier, B.; Yim, T.; Lee, K. T.; Bein, T.; Nazar, L. F. Angew. Chem. 2012, 124, 3651.

[39] Kim, J.; Lee, D. J.; Jung, H. G.; Sun, Y.-K.; Hassoun, J.; Scrosati, B. Adv. Funct. Mater. 2012, DOI: 10.1002/adfm.201200689.

[40] He, G.; Ji, X.; Nazar, L. Energy Environ. Sci. 2011, 4, 2878.

[41] Jayaprakash, N.; Shen, J.; Moganty, S. S.; Corona, A.; Archer, L. A Angew. Chem. 2011, 123, 6026.

[42] Zhang, C.; Wu, H. B.; Yuan, C.; Guo, Z.; Lou, X. W. Angew. Chem. 2012, 124, 9730.

[43] Liang, C.; Dudney, N. J.; Howe, J. Y. Chem. Mater. 2009, 21, 4724.

[44] Zhang, B.; Qin, X.; Li, G. R.; Gao, X. P. Energy Environ. Sci. 2010, $3,1531$.

[45] Cao, Y.; Li, X.; Aksay, I. A.; Lemmon, J.; Nie, Z.; Yang, Z.; Liu, J. Phys. Chem. Chem. Phys. 2011, 13, 7660.

[46] Ji, L.; Rao, M.; Zheng, H.; Zhang, L.; Li, Y.; Duan, W.; Guo, J.; Cairns, E. J.; Zhang, Y. J. Am. Chem. Soc. 2011, 133, 18522.

[47] Li, S.; Xie, M.; Liu, J.; Wang, H.; Yan, H. Electrochem. Solid-State Lett. 2011, 14, A105.

[48] Wang, H.; Yang, Y.; Liang, Y.; Robinson, J. T.; Li, Y.; Jackson, A.; Cui, Y.; Dai, H. Nano Lett. 2011, 11, 2644.

[49] Wang, J. Z.; Lu, L.; Choucair, M.; Stride, J. A.; Xu, X.; Liu, H. K. J. Power Sources 2011, 196, 7030.

[50] Evers, S.; Nazar, L. F. Chem. Commun. 2012, 48, 1233.

[51] Li, N.; Zheng, M.; Lu, H.; Hu, Z.; Shen, C.; Chang, X.; Ji, G.; Cao, J.; Shi, Y. Chem. Commun. 2012, 48, 4016.

[52] Park, M. S.; Yu, J. S.; Kim, K. J.; Jeong, G.; Kim, J. H.; Jo, Y. N.; Hwang, U.; Kang, S.; Woo, T.; Kim, Y. J. Phys. Chem. Chem. Phys. 2012, 14, 6796 .

[53] Sun, H.; Xu, G. L.; Xu, Y. F.; Sun, S. G.; Zhang, X.; Qiu, Y.; Yang, S. 
Nano Res. 2012, 10, 726 .

[54] Wang, D. W.; Zhou, G.; Li, F.; Wu, K. H.; Lu, G. Q.; Cheng, H. M.; Gentle, I. R. Phys. Chem. Chem. Phys. 2012, 14, 8703.

[55] Wang, Y. X.; Huang, L.; Sun, L. C.; Xie, S. Y.; Xu, G. L.; Chen, S. R.; Xu, Y. F.; Li, J. T.; Chou, S. L.; Dou, S. X.; Sun, S. G. J. Mater. Chem. 2012, 22, 4744.

[56] Wei, Z. K.; Chen, J. J.; Qin, L. L.; Nemage, A. W.; Zheng, M. S.; Dong, Q. F. J. Electrochem. Soc. 2012, 159, A1236.

[57] Yan, Y.; Yin, Y. X.; Xin, S.; Guo, Y. G.; Wan, L. J. Chem. Commun. 2012, 48, 10663.

[58] Zhang, F. F.; Zhang, X. B.; Dong, Y. H.; Wang, L. M. J. Mater. Chem. 2012, 22, 11452.

[59] Zhang, L.; Ji, L.; Glans, P. A.; Zhang, Y.; Zhu, J.; Guo, J. Phys. Chem. Chem. Phys. 2012, 14, 13670.

[60] Huang, J. Q.; Liu, X. F.; Zhang, Q.; Chen, C. M.; Zhao, M. Q.; Zhang, S. M.; Zhu, W.; Qian, W. Z.; Wei, F. Nano Energy 2012, DOI: 10.1016/j.nanoen.2012.10.003.

[61] Elazari, R.; Salitra, G.; Garsuch, A.; Panchenko, A.; Aurbach, D. Adv. Mater. 2011, 23, 5641.

[62] Wang, J.; Yang, J.; Xie, J.; Xu, N. Adv. Mater. 2002, 14, 962.

[63] Wang, J.; Yang, J.; Wan, C.; Du, K.; Xie, J.; Xu, N. Adv. Funct. Mater. 2003, 13, 487.

[64] Yu, X.; Xie, J.; Li, Y.; Huang, H.; Lai, C.; Wang, K. J. Power Sources 2005, 146, 335.

[65] Yin, L.; Wang, J.; Yang, J.; Nuli, Y. J. Mater. Chem. 2011, 21, 6807.

[66] Yin, L.; Wang, J.; Lin, F.; Yang, J.; Nuli, Y. Energy Environ. Sci. 2012, 5, 6966.

[67] Yin, L.; Wang, J.; Yu, X.; Monroe, C. W.; NuLi, Y.; Yang, J. Chem. Commun. 2012, 48, 7868 .

[68] Wang, J.; Chen, J.; Konstantinov, K.; Zhao, L.; Ng, S. H.; Wang, G. X.; Guo, Z. P.; Liu, H. K. Electrochim. Acta 2006, 51, 4634.

[69] Sun, M.; Zhang, S.; Jiang, T.; Zhang, L.; Yu, J. Electrochem. Commun. 2008, 10, 1819.

[70] Qiu, L.; Zhang, S.; Zhang, L.; Sun, M.; Wang, W. Electrochim. Acta 2010, 55, 4632.

[71] Liang, X.; Liu, Y.; Wen, Z.; Huang, L.; Wang, X.; Zhang, H. J. Power Sources 2011, 196, 6951.

[72] Liang, X.; Wen, Z.; Liu, Y.; Wang, X.; Zhang, H.; Wu, M.; Huang, L. Solid State Ionics 2011, 192, 347.
[73] Liang, X.; Wen, Z.; Liu, Y.; Zhang, H.; Jin, J.; Wu, M.; Wu, X. J. Power Sources 2012, 206, 409.

[74] Fu, Y.; Manthiram, A. RSC Adv. 2012, 2, 5927.

[75] Fu, Y.; Manthiram, A. Chem. Mater. 2012, 24, 3081.

[76] Fu, Y.; Su, Y. S.; Manthiram, A. J. Electrochem. Soc. 2012, 159, A1420.

[77] Zhang, Y.; Bakenov, Z.; Zhao, Y.; Konarov, A.; Doan, T. N. L.; Malik, M.; Paron, T.; Chen, P. J. Power Sources 2012, 208, 1.

[78] Wu, F.; Wu, S.; Chen, R.; Chen, J.; Chen, S. Electrochem. Solid-State Lett. 2010, 13, A29.

[79] Wu, F.; Chen, J.; Chen, R.; Wu, S.; Li, L.; Chen, S.; Zhao, T. J. Phys. Chem. C 2011, 115, 6057 .

[80] Wu, F.; Chen, J.; Li, L.; Zhao, T.; Chen, R. J. Phys. Chem. C 2011, $115,24411$.

[81] Li, G. C.; Li, G. R.; Ye, S. H.; Gao, X. P. Adv. Energy Mater. 2012, 2 , 1238.

[82] Xiao, L.; Cao, Y.; Xiao, J.; Schwenzer, B.; Engelhard, M. H.; Liu, J. Adv. Mater. 2012, 24, 1176

[83] Yang, Y.; Yu, G.; Cha, J. J.; Wu, H.; Vosgueritchian, M.; Yao, Y.; Bao, Z.; Cui, Y. ACS Nano 2011, 5, 9187.

[84] Song, M. S.; Han, S. C.; Kim, H. S.; Kim, J. H.; Kim, K. T.; Kang, Y. M.; Ahn, H. J.; Dou, S. X.; Lee, J. Y. J. Electrochem. Soc. 2004 151, A791.

[85] Zheng, W.; Hu, X. G.; Zhang, C. F. Electrochem. Solid-State Lett. 2006, 9, A 364.

[86] Zhang, Y.; Wu, X.; Feng, H.; Wang, L.; Zhang, A.; Xia, T.; Dong, H. Int. J. Hydrogen Energy 2009, 34, 1556.

[87] Evers, S.; Yim, T.; Nazar, L. F. J. Phys. Chem. C 2012, 116, 19653.

[88] Zhang, Y.; Bakenov, Z.; Zhao, Y.; Konarov, A.; Doan, T. N. L.; Sun, K. E. K.; Yermukhambetova, A.; Chen, P. Powder Technol. 2013, $235,248$.

[89] Zhang, Y.; Zhao, Y.; Yermukhambetova, A.; Bakenov, Z.; Chen, P. J. Mater. Chem. A 2013, 1, 295.

[90] Lee, K. T.; Black, R.; Yim, T.; Ji, X.; Nazar, L. F. Adv. Energy Mater 2012, 2, 1490.

[91] Cakan, R. D.; Morcrette, M.; Nouar, F.; Davoisne, C.; Devic, T.; Gonbeau, D.; Dominko, R.; Serre, C.; Tarascon, J. M. J. Am. Chem. Soc. 2011, 133, 16154. 